\title{
BMJ Open Barriers and facilitators to interdisciplinary communication during consultations: a qualitative study
}

\author{
Pingyang Liu, ${ }^{1}$ Audrey Lyndon (D) , ${ }^{2}$ Jane L Holl, ${ }^{3}$ Julie Johnson, ${ }^{4,5}$ \\ Karl Y Bilimoria, ${ }^{4,5}$ Anne M Stey (1) ${ }^{4,5}$
}

To cite: Liu P, Lyndon A, Holl JL, et al. Barriers and facilitators to interdisciplinary communication during consultations: a qualitative study. BMJ Open 2021;11:e046111. doi:10.1136/ bmjopen-2020-046111

- Prepublication history and online supplemental material for this paper is available online. To view these files, please visit the journal online. (http://dx.doi. org/10.1136/bmjopen-2020046111).

Received 11 December 2020 Accepted 06 July 2021

Check for updates

(c) Author(s) (or their employer(s)) 2021. Re-use permitted under CC BY-NC. No commercial re-use. See rights and permissions. Published by BMJ.

${ }^{1}$ Department of Surgery, University of California San Francisco, San Francisco, California, USA

${ }^{2}$ Rory Meyers College of Nursing, New York University, New York City, New York, USA ${ }^{3}$ Department of Neurology, University of Chicago, Chicago, Illinois, USA

${ }^{4}$ Center of Health Services and Outcomes Research, Institute for Public Health and Medicine, Chicago, Illinois, USA

${ }^{5}$ Department of Surgery, Northwestern University Feinberg School of Medicine, Chicago, Illinois, USA

Correspondence to

Dr Anne M Stey;

as013j@gmail.com

\section{ABSTRACT}

Objective Communication failures between clinicians lead to poor patient outcomes. Critically injured patients have multiple injured organ systems and require complex multidisciplinary care from a wide range of healthcare professionals and communication failures are abundantly common. This study sought to determine barriers and facilitators to interdisciplinary communication between the consulting trauma, intensive care unit (ICU) team and specialty consultants for critically injured patients at an urban, safety-net, level 1 trauma centre.

Design An observational qualitative study of barriers and facilitators to interdisciplinary communication.

Setting We conducted observations of daily rounds in two trauma surgical ICUs and recorded the most frequently consulted teams.

Participants Key informant interviews after presenting clinical vignettes as discussion prompts were conducted with a broad range of clinicians from the ICUs and physicians and nurse practitioners from the consultant teams who were identified during the observations. Interviews were recorded and transcribed verbatim. Data of these 10 interviews were combined with primary transcript data from prior study (25 interviews) and analysed together because of the same setting with same themes. Independent coding of the transcripts, with iterative reconciliation, was performed by two coders.

Outcomes measures Facilitators and barriers of interdisciplinary communication were identified. Results A total of 35 interview transcripts were analysed. Cardiology and interventional radiology were the most frequently consulted teams. Consulting and consultant clinicians reported that perceived accessibility from the team seeking a consultation and the consultant team impacted interdisciplinary communication. Accessibility had a physical dimension as well as a psychological dimension. Accessibility was demonstrated by responsiveness between clinicians of different disciplines and in turn facilitated interdisciplinary communication. Social norms, cognitive biases, hierarchy and relationships were reported as both facilitators and barriers to accessibility, and therefore, interdisciplinary communication.

Conclusion Accessibility impacted interdisciplinary communication between the consulting and the consultant team.

\section{Strengths and limitations of this study}

- This study compiles qualitative interviews from a broad range of health professionals (nurses, respiratory therapists, neurologists, neurosurgeons, anaesthesiologists, cardiologists, interventional radiologists, trauma surgeons and intensivists) to gain insight into interdisciplinary communication.

- This study triangulated observational and interview data to understand the nature of professional relationship.

- This study probed complex organisational behaviour topics in the fast pace and stressful environment of caring for people with critical injury.

- This study is a single-centre study and observations are subject to local context.

- This study does not determine how to recognise, teach and promote accessibility.

Article summary Elucidates barriers and facilitators to interdisciplinary communication between consulting and consultant teams.

\section{INTRODUCTION}

Critically injured patients typically present with injuries to multiple organ systems, and therefore, require care from multiple clinician specialists from a range of disciplines with diverse backgrounds. ${ }^{1}$ Shared mental models and situational awareness can facilitate team-based care provided by clinicians with different backgrounds and experiences. ${ }^{2}$ Generating shared mental models and situational awareness requires effective interdisciplinary communication. ${ }^{3}$ When critically injured patients with multiple injured organ systems are admitted, requesting consultations by specialists is the means of formally engaging clinicians from another discipline. There can be rapid formation and dissolution of ad hoc teams from multiple disciplines, depending on the specific clinical circumstances. Interdisciplinary teams caring for critically injured patients typically include 
trauma surgeons, surgical intensivists, intensive care nurses, pharmacists, respiratory therapists, neurosurgeons, orthopaedic surgeons, cardiologists, anaesthesiologists, interventional radiologists, neurologists, plastic surgeons, dieticians and physical therapists. However, different backgrounds can contribute to professional culture clash, which can impair team performance and result in adverse events for patients. ${ }^{45}$

Communication among clinicians about the care of the critically injured patient frequently breaks down and leads to poor outcomes. ${ }^{6-11}$ Identifying barriers and facilitators to interdisciplinary communication is necessary to develop targeted interventions to promote highquality interdisciplinary communication, generate shared mental models and situational awareness and improve team-based surgical critical care. This study concerns team-to-team communication. This exploratory qualitative study sought to identify the barriers and facilitators to interdisciplinary communication during specialty consultations for critically injured patients.

\section{METHODS}

\section{Conceptual model}

The Leonard Model ${ }^{12}$ was used as the underlying framework onto which subsequent modifications were made by the research team to create a conceptual model of the flow of interdisciplinary communication during teambased care of critically injured surgical patients (online supplemental appendix 1). The model was iteratively expanded on by emailing the model graphic to 10 nonparticipant clinicians, specifically intensive care unit (ICU) nurses, respiratory therapists, trauma surgeons, neurologists, orthopaedic surgeons, neurosurgeons, anaesthesiologists, radiologists and critical care intensivists, and soliciting feedback through written edits to the model. This resulting modified model was used to inform the development of the semistructured interview guide.

\section{Setting}

The study was conducted in two Trauma Surgical ICUs in a level 1 trauma centre at an urban, safety-net hospital. The ICUs are state-of-the-art, 16 -bed units, which provide care for patients with all types of traumatic injuries. The ICUs are hybrid open units, with the Trauma team typically being the primary team that manages the patients. The trauma team writes most daily orders and requests specialty consultations. The nurse-to-patient staffing ratio is $1: 2$. There are one or two respiratory therapists per 16 bed-unit, depending on the number of ventilated patients. The US employs Registered Respiratory Therapist who are specialised healthcare practitioners trained in pulmonary medicine to work under the direction of physicians to perform pulmonary hygiene and airway clearance therapy and implement changes to the noninvasive and invasive mechanical ventilation in people suffering from acute critical conditions, cardiac and pulmonary disease. Respiratory therapists on completion of their graduate degree as well as national written and a clinical simulation examination administered by the National Board for Respiratory Care, initiate and manage pulmonary life support for people in ICUs and emergency departments under the supervision of physicians. There is one critical-care pharmacist for both units. The study period was from October 2017 to September 2019.

\section{Observations}

A PhD biomedical researcher conducted a series of nonparticipant observations of Trauma and ICU Team daily rounds to identify the most commonly requested specialty consultations by the ICU and trauma teams. Field notes were used to document the observations and the observer debriefed with the lead investigator immediately after each observation. ${ }^{13}$

\section{Semistructured interviews \\ Cohort description}

Purposive sampling was used to recruit attending physician consultants on staff at the hospital. Participants were approached by email. Interviews were conducted in a private space at the participants' workplace. Each interview was planned to last approximately $30 \mathrm{~min}$. This study builds on the research team's research about interdisciplinary team care for critically injured patients. Previously, key informant interviews in the same two ICUs revealed the importance of interdisciplinary communication for decision making. ${ }^{3}$ Comments about specialty consultations were outside the scope of the prior study about interdisciplinary professionals' interactions in clinical decision making. We combined the primary transcript data from the prior 25 interviews with the new data from structured vignette interviews to conduct an analysis of the facilitators and barriers of interdisciplinary communication. Methods of the prior study are detailed elsewhere. ${ }^{3}$

\section{Patient and public involvement}

The development of the research question and outcome measures were informed by patients' priorities, experience and preferences because interdisciplinary communication failures are a common cause of adverse patient events. Patients were not involved in the design of this study. Patients were not involved in the recruitment nor the conduct of this study. The results will be disseminated to study participants on acceptance by a follow-up email with an executive summary.

\section{Interview development}

The research team created and pilot tested interview guide with diverse non-participant clinicians, representing the disciplines, professions and professional levels in the ICU (online supplemental appendix 2). A male PhD-level researcher (PL) and a female trauma surgeon (AMS), both with prior qualitative research training and expertise, conducted the interviews. Participants were informed that the aim of the research was to understand communication between clinicians. 
At the beginning of the interview, participants were presented with the appended clinical vignettes that had been created and iteratively refined by a group of non-participant trauma surgeons, radiologists, intensivists and cardiologists (online supplemental appendix 3). One vignette depicted a patient with a well-defined question for the specialist consultant for which his/her opinion and recommendations were indispensable to the patient's care. Another vignette depicted a patient with a complicated presentation wherein the specialist was consulted without a clearly defined question being asked. After reading each vignette, participants were asked questions to elicit their perspectives about interdisciplinary communication in the context of each consultation. Four questions focused on the timing and decision-making process of requesting a consultation and four questions focused on feedback and updates following a consultation. Interviews were done until data saturation was reached. The interviews were audio recorded, and transcribed verbatim with redaction of all identifying information and combined with field notes taken during interviews and observation. Transcripts were returned to participants for correction.

\section{Analysis}

We analysed the data thematically following the methods of Braun and Clarke. ${ }^{14-16}$ ATLAS.ti, ATLAS.ti Scientific Software Development, was used to manage the transcribed interviews and codes. Coding and theme identification was performed independently by two coders (PL and AMS). Discrepancies were addressed with iterative revisions to reach consensus. Attention was given to both semantic or surface and latent or underlying meaning expressed in transcripts. Thematic analysis was conducted through coding, category formulation, and sorting for data reduction and thematic structuring of the data. First a code book was developed after reviewing all transcripts by the two coders. Once the code book was developed, the coders and a PhD-level qualitative methodologist (AL), specialised in interdisciplinary communication, supervised category formulation. Sorting for data reduction was done independently by the coders and the sorting was then reviewed and disagreements discussed until consensus was reached over videoconference calls with supervision of qualitative methodologists. Thematic structuring and the subsequent development of the central theme and subthemes was performed through joint virtual sessions between all authors. The coding tree consisted of a central theme of accessibility and four latent subthemes of social norms, cognitive biases, hierarchy and relationships. All themes were derived from the data. Three of the authors, PL, AMS and AL, reviewed the transcripts and selected quotes that elaborated the themes the most eloquently and poignantly.

\section{RESULTS}

Thirty-five interview transcripts were included in the analysis. Ten from the current and 25 from the prior set of

\begin{tabular}{lc}
\hline Table 1 Participant sample characteristics & \\
\hline Sample characteristics & $\mathbf{N}(\%)$ \\
\hline Age & \\
\hline $18-30$ & $2(5.7)$ \\
$30-45$ & $12(34.3)$ \\
$46-60$ & $17(48.6)$ \\
$>61$ & $4(11.4)$ \\
\hline Female gender & $19(54.3)$ \\
\hline Years of experience & \\
\hline$<5$ & $5(15.3)$ \\
\hline $6-15$ & $15(42.9)$ \\
\hline $16-25$ & $12(34.3)$ \\
$>26$ & $3(8.6)$ \\
\hline
\end{tabular}

interviews. Table 1 shows the demographic and professional participant characteristics.

Figure 1 shows the most consulted specialties based on the observations conducted.

\section{Central theme: accessibility}

Participants described accessibility as having both physical and psychological component. Physical accessibility was influenced by the structural factors such as physical space, rounding schedules, and clinician presence elsewhere than in the ICU (eg, operating room). Rounding schedules were reported to influence perceived accessibility and which differed considerably, based on a clinician's role. As a result, there were few opportunities for an interdisciplinary team to meet at a patient's bedside. For example, specialist consultant clinicians who had prior experience working at different institutions noted the benefits of sharing physical space in a joint ICU.

[In the other hospital] the ICU [was shared] and the teams would be there, [be]cause we all have patients in there. So, if Trauma team was over here and I was seeing a patient over there, I'd walk by and, 'Hey, what do you got? You got something good?' [It was] the space, the design of where you work. Cardiologist

[At my former workplace] We just joined the rounds, [that doesn't happen here]. We did it whether or not we were called to join the rounds, and that was very valuable for us as well as everybody else, [be]cause somebody would bring up something and I could contribute.' Cardiologist

Other reported structural factors influencing physical accessibility included difficulty in identifying the appropriate individual to contact on the team requesting a consultation (consulting team). Furthermore, reaching the appropriate colleague when pages and calls were not answered or not answered in a timely fashion were another identified barrier. Finally, there were professionspecific barriers such as surgeons being in the operating room for lengthy periods of time. 


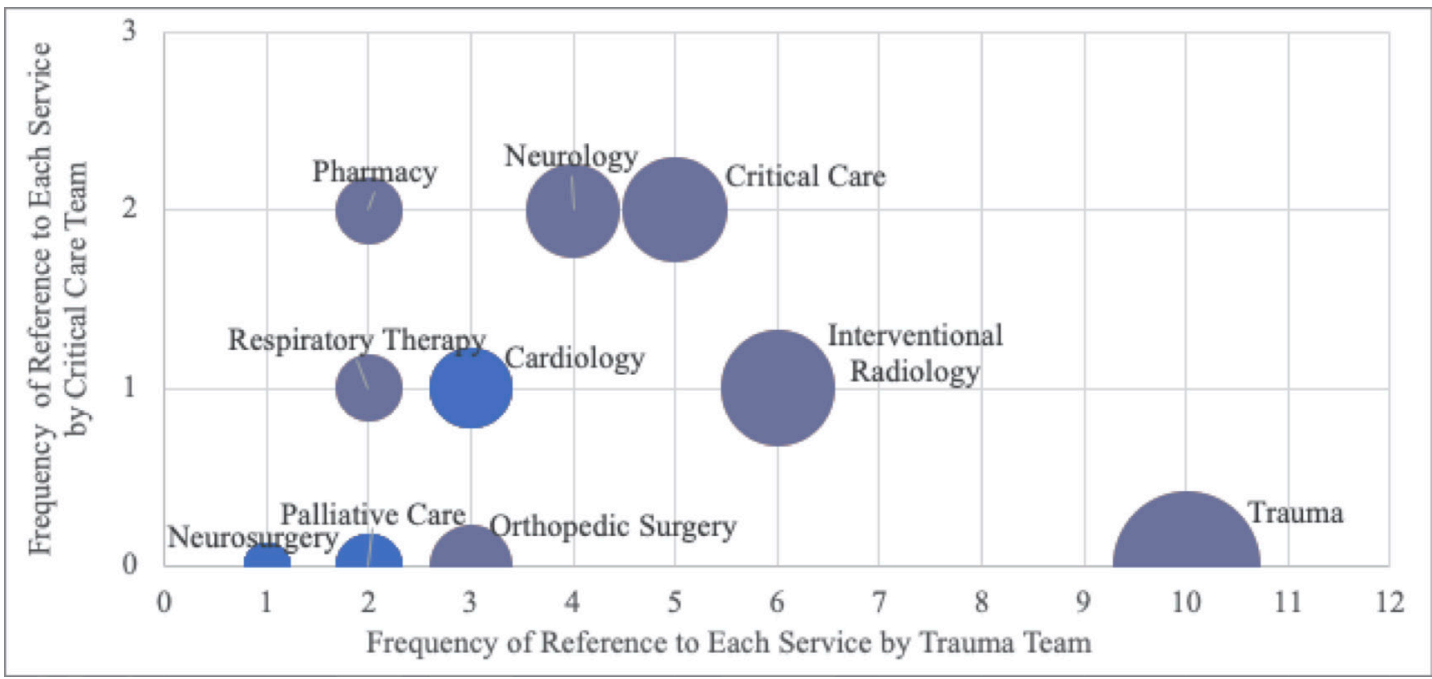

Figure 1 Bubble plot of interdisciplinary clinicians most frequently sought out for consultation for critically injured patients. The $y$-axis depicts frequency of reference to a professional service by any member of the intensivists' critical care team during critical care rounds. The $\mathrm{x}$-axis depicts the frequency of reference to a professional service by any member of the trauma surgery team during trauma surgery team rounds. Interteam and intrateam references are depicted. The size of the bubble demonstrates the sum volume of references. The two professional services most frequently referenced and not included in previous study on how interdisciplinary decisions are made were the cardiology and interventional radiology teams.

Figuring out who to contact is usually a challenge. It's about as fun as taking this pen and sticking it through my eyeball. Cardiologist

Trauma is one case. Neuro's a little different, they seek us out at higher levels, and they're not in the OR all the time. Cardiologist

Participants reported that perceived psychological accessibility was demonstrated by 'responsiveness.' Consulting teams and consultant teams have different mental models of responsiveness. Responsiveness from the consulting team perspective was described by the consultants' willingness to perform the consultation. Conversely, responsiveness from the consultants' perspective was described as (1) how the consult was framed and (2) willingness of the consulting team to engage with consultants. Consultant team members perceived some requests as a request for the consultant to fulfil a role of 'technician' rather than for the consultant's clinical expertise.

It would be nice to have a discussion like 'Hey, I'm concerned about this clinically. How can I answer this question?' Rather than, 'Hey, I want this procedure done. Cardiologist [Consultant perspective]

People will listen if it is phrased in a question rather than phrased in a 'I think we should do this' kind of way. Part of that reflects respect for the people who are invested, but also part of it reflects the power differential. Trauma Surgeon[Consulting Team's Perspective]

Consultant teams reported the consulting team as engaged with them in one of two ways. First, the consulting team could simply follow the consultants' recommendations. Second, the consulting team could directly discuss the patient with the consultant team. These discussions allowed for explicit sharing of specialty specific data to build a shared mental model.

Let's have that discussion, and then everybody learns a little bit more about how the other teams work and we have some interdisciplinary team-building because we've had these conversations. Neurosurgeon

Teams who engaged and were seen as responsive and therefore accessible, created shared mental models and situational awareness more easily. For example, shared recognition by trauma surgeons and neurosurgeons of a worsening neurological exam in a patient with traumatic brain injury would ideally lead to discussions creating a shared mental model of the suspected cause, increased intracranial pressure, and situational awareness, such as the thresholds for escalating care.

Neurosurgery and Trauma work well together in the sandbox in the way of aligning plans pretty quickly and being able to be accessible during the day for like if plans change or if things are not going well. Neurosurgeon

I think there is an opportunity for better communication again between all the teams. When we've gotten to hour 12 with this [patient], now these are the things I think that would mitigate these oxygenation issues, what are our thresholds for ECMO today, what are we going to have a short leash on and what do we think she's going to be able to ride out and survive? Respiratory Therapist

When there was a lack of responsiveness, both the consulting and consultant teams reported it was more difficult to build shared understanding the patient's 


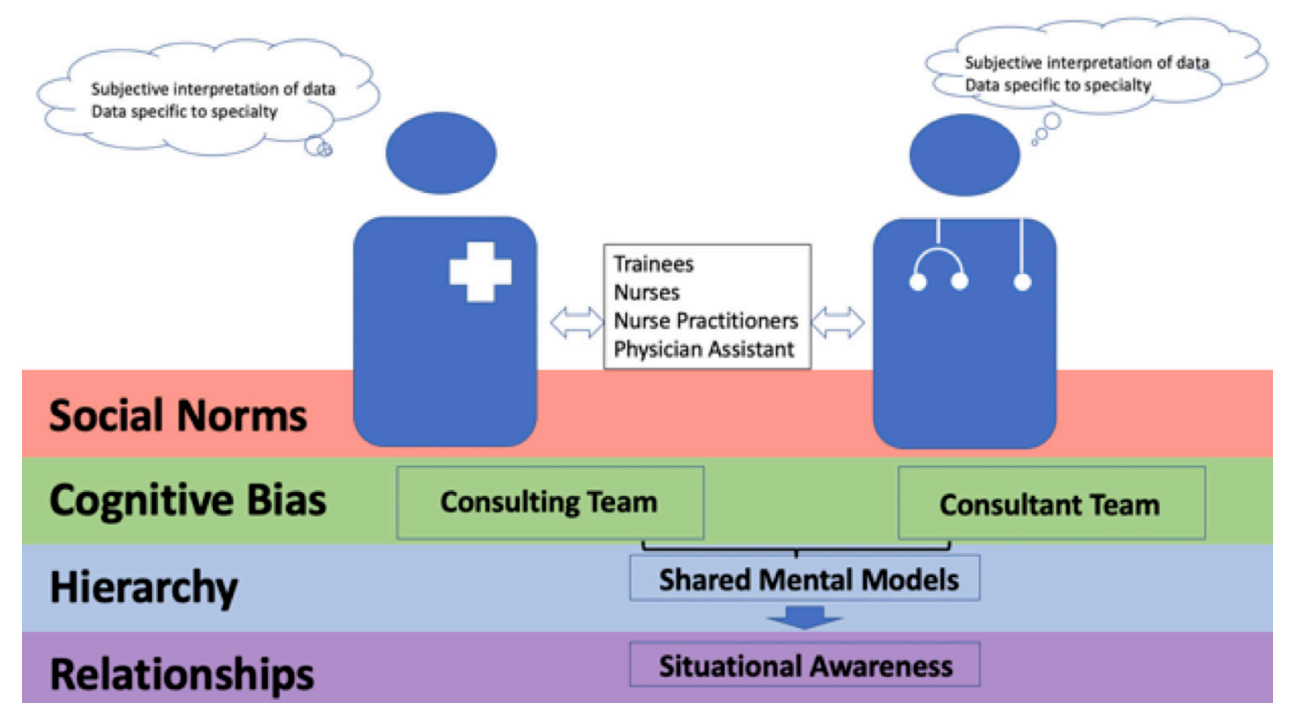

Figure 2 Physical and psychological accessibility were indispensable to interdisciplinary communication. The central theme of accessibility between consulting and consultant is depicted as arrows to and from the consulting and consultants, with trainees, nurses, nurse practitioners and physicians' assistants in the middle. Latent subthemes social norms, cognitive bias, hierarchy and relationships that served as barriers and facilitators are labelled as shaded background through which shared mental models and situational awareness must be developed.

problem and how to treat it. Instead each team retreated into their own mental model, based on their frame of reference and clinical experience. Objective clinical data could be interpreted differently by each team. For example, sinus tachycardia in an injured patient may be interpreted as a sign of haemorrhagic shock by a trauma team, whereas, a cardiology team may be concerned for a pulmonary embolus. Yet, there was little recourse, by either team, to demand responsiveness or accessibility to create a shared mental model other than escalating the question to the clinicians at the top of the hierarchy of either team.

It is not the case that the surgical resident or attending will listen or will take the recommendation. Most of the time, as far as we can tell, they don't. Cardiologist

I've presented a very logical option for them, and [they respond], 'No, I want this.' 'I want this' doesn't mean anything to me. I want lots of things in life. Radiologist

Accessibility was reported to be influenced both positively and negatively by four latent themes: social norms, hierarchy, cognitive biases and relationships. (figure 2).

\section{Subtheme: social norms}

Social norms were described as shaping interactions between different teams. These social norms ranged from 'unspoken' conventions between the consultant and consulting teams (eg, pelvic fracture) to explicit management protocols. In both, unspoken conventions and management protocols detailed the timing and extent of involvement of specialty consultants (eg, spinal cord injury protocols).
Yeah, [communication] is very smooth-it's basically the standard pelvic fracture patient that we always treat. Radiologist

To level the playing field, you say this is what we do for a spinal cord injury patient regardless of whose service you are on and that's the reason the spinal cord injury guidelines were created. Neurologist

\section{Subtheme: hierarchy}

Clinicians reported that hierarchy influenced with whom they were expected to interact, and served as a barrier to effective communication when the flow of information depended on a sole pathway to the team leader, or attending physician. If any person along the pathway to the leader did not pass along the information, and the other team members earlier in the pathway did not communicate directly with the leader, then the information never reached the leader. A common example was described: An ICU nurse, typically with many years of clinical experience, recognises the early signs of patient deterioration and transmits the information to a resident, who fails to pass the information along to the senior resident, who in turn, never informs the attending.

The nurses don't want to go past the residents to the attendings because they feel it's violating the chain of command. Trauma Surgeon

I know it's important for interns and residents to learn, but when you can't make a decision, then you need to talk to somebody above you in a situation like this. Trauma ICU Nurse

Hierarchy was also described as a facilitator of accessibility when communications were escalated to clinicians at higher levels. This escalation suggested clinical urgency 
and quickly led to agreement of a shared mental model. Interactions between clinicians at a higher level signalled the importance of the information.

When I feel like something is more urgent, I skip the junior level people and then I speak to whomever the attending is. Cardiologist

If the attending talks to me and [they] feel that strongly about it, the fact that you're even calling me up and talking to me about it says that this is important to them. If it's that important to them, at least for now, I'll probably go along. Trauma Surgeon

\section{Subtheme: cognitive bias}

Cognitive biases influenced accessibility and therefore interdisciplinary communication both positively or negatively. Demographic characteristics of a clinician (either consultant or consulting) were associated with cognitive biases. For example, young clinicians and surgeons were perceived as less capable and more difficult to interact with.

Young attendings, frankly they don't have the comfort with this. Radiologist

Some of the surgeons have personalities. You know that. Anesthesiologist

Cognitive bias was also reported in relation to clinical presentations. Consultant teams were reported as interpreting objective clinical data somewhat subjectively, based on the specialty's frame of reference and clinical experience. For example, most intensivists consider an undrained pleural effusion as a potential source of sepsis requiring drainage because they care for patients with parapneumonic effusions regularly. However, an interventional radiologist, who frequently places a drainage catheter of large uncomplicated effusions, may find that drainage of a small serous effusion that may or may not be infected, to be less compelling. These different experiences were described to lead to conflict, or 'professional culture clash.'

I think the best thing is when it is actual objective data that's being presented. The problem is that very often [interpretation] is a subjective well. Radiologist

Communication can be frustrating with somebody who just actually doesn't have the knowledge depth and experience to have that anticipation of badness coming with a patient. Trauma ICU Nurse

Explicitly stating these differences in interpretations allowed for conflicts to be overcome. For example, explicitly stating that a patient is in septic shock in the absence of another clear infection source and that a small serous pleural effusion seems the most likely source could help build a shared mental model between an intensivist and a radiologist.

Cognitive biases could also emerge from previous experiences with certain individuals or services. Cognitive biases were reported to act as a facilitator to accessibility and therefore interdisciplinary communication if there was previously perceived reciprocity from an individual or service. Cognitive biases were perceived to act as a barrier when the consultant team assumed or previously perceived ulterior motives for the consultation request. For example, if a surgeon insisted that a radiologist perform a procedure, despite the radiologist's previous assertion that the procedure was not feasible or indicated, then the surgeon was perceived as difficult, behaving as if they knew how to perform the radiologist's job better than the radiologist. If someone was labelled as difficult, the cognitive bias persisted as a barrier for future interactions. These cognitive biases risked setting the tone for positive or negative working relationships.

I've been here 40 years, and there are people I know on Surgery that I would do anything for and they would do the same for me. Cardiologist

Other specialties believe that they are better at interpreting those studies than the people who are specialized in doing that and that's foolish. Radiologist

Cardiology is not infrequently being consulted in order to get a decent history and physical on the record and I don't like that. We want these guys to do their work. Cardiologist

\section{Subtheme: relationships}

Relationships was reported to positively or negatively impact accessibility and, therefore, interdisciplinary communication. Relationships were built by previously working together, clinically or otherwise. For example, working on quality improvement panels or in morbidity and mortality conferences created opportunities for professional interactions, outside of patient care activities. Feeling respected and heard in other working relationships created positive relationships and facilitated accessibility and therefore interdisciplinary communication.

The trauma team is probably [most responsive] because we all work-in the OR, we work closely. It's a lot easier to communicate and discuss. And neurosurgery and neurology is different mindset. It's a little bit difficult. Anesthesiologist

I think we really try to do these by consensus because we want everyone who is taking care of the patient to want to feel they're being heard about what they think is appropriate or inappropriate. And also, at the same time, even if they don't necessarily agree with the final decision, medical care plan, that they at least have been heard and that their opinions have been considered Neurosurgeon

Conversely, newcomers who lacked previous interactions and positive relationships were reported to struggle. Relationships served as barriers to accessibility and interdisciplinary communication. This was particularly true when negative relationships existed because individuals had felt previously disrespected or threatened in prior interactions. Disagreements between two individuals were 
reported to lead to assumptions by one individual that the other lacked respect for their clinical opinion. Such assumptions reportedly created defensiveness, posturing and inflexibility in interactions.

Every now and then you'll get individuals that speak up or raise their voice or use bad body language, and not so respectful of each other's professional [capacities] Respiratory Therapist

Some teams feel threatened. Threatened in terms of the decisions that this is how we do things and I don't need you to tell me what I should or should not be doing because I have done this. Neurologist

Defensiveness, posturing and inflexibility due to differences in clinical impressions, clinical management styles, and poor communication of those management styles, led to failure in reaching shared mental models and further impeded future accessibility and interdisciplinary communication. For example, a neurologist who believes in weaning external ventricular drains and a neurosurgeon who believes in simply clamping and removing the drain may never reach consensus on patient management. In future interactions, the neurosurgeon may not even involve the neurologist or follow their recommendations.

[People] are agitated when they really do not feel respected and heard, whether that is because somebody is not respecting them or because they just are not able to say clinically what their concerns are and then we cannot address them. Neurosurgeon

\section{DISCUSSION}

This study was designed to identify the barriers and facilitators to interdisciplinary communication between a trauma consulting team that is primarily responsible for a critically injured patient's care and specialty consultant teams. The study showed that interdisciplinary communication for critically injured patients was highly dependent on perceived accessibility of both the consulting and consultant teams. Responsiveness was a facilitator to accessibility and therefore interdisciplinary communication. When sufficient responsiveness by either team was present, perceived accessibility increased and barriers were more easily overcome to create shared mental models and situational awareness. Lack of responsiveness reinforced barriers to interdisciplinary communication and impaired formation of shared mental models. Social norms, hierarchy, cognitive bias and relationships functioned as both barriers and facilitators to accessibility and therefore interdisciplinary communication.

These findings echo the findings in the team science literature. Clinical medicine has grown in breadth of knowledge and has become more specialised. Healthcare systems have become highly dependent on teams of specialists to provide interdisciplinary team care. ${ }^{17}$ Yet, engagement of clinicians from multiple disciplines, with vastly different clinical backgrounds and professional culture in the setting of increased time pressure and persistent power differentials, has been challenging. ${ }^{18}$ Professional culture clash occurs when teams with different work schedules, jargon and working styles must come together to solve a problem. ${ }^{19}$ Interdisciplinary rounds have been used for over a decade with mixed results. ${ }^{20}$ The findings of this study suggest that merely being present at rounds, or physical accessibility, is insufficient for interdisciplinary communication to occur. Each member of every team needs to feel respected and heard. These findings illustrate the incomplete acceptance in healthcare of the importance of psychological safety, as clinicians still report hierarchy, social norms and negative relationships as barriers to effective communication and high level team performance. ${ }^{21}$ What this study adds is that relationships, hierarchy, cognitive bias and social norm affect communication context by impacting psychological accessibility, and therefore, interdisciplinary communication in a wide range of ways from positive (acting as a facilitator) to negative (acting as a barrier).

We interpret our findings of responsiveness as a facilitator to accessibility as a means of creating psychological safety. Other barriers we identified are well-known threats to psychological safety including hierarchy ${ }^{19}$ and cognitive bias. ${ }^{22}$ Engaged responsiveness may be a means of overcoming these barriers to interdisciplinary communication that threaten psychological safety. Recognising, teaching and promoting responsiveness requires more study.

This study has several limitations. First, although clinicians from different disciplines and different professional levels were interviewed, neither the perspectives of all disciplines nor of all professional levels were captured. Although trainees (resident doctors, medical, nursing and respiratory therapy students) were not included in this study, there was quite a bit of discussion about them from the practising fully trained staff. We found these topics to be too complex and different from those of just communication between different professional service disciplines. Trainee role in communication is an important future direction. Second, teams were cognizant that study staff were observing rounds for research purposes. This awareness may have led them to deviate from normal routine. However, teams appeared to acclimate quickly to the observer's presence. Third, the study setting was limited to two ICUs in a single trauma centre, which limits generalisability of the findings. However, the purpose of this qualitative study was to demonstrate transferability, identifying what is potentially relevant to other settings, rather than generalisability. ${ }^{23}$ Transferability occurs whenever a person or group in one setting considers adopting something from another that the research has identified. The consistency of our findings regarding communication context, relationships and cognitive bias with existing literature suggest that our findings are likely transferable to other settings. ${ }^{22} 2425$ Further 
study is needed to determine how to recognise, teach and promote responsive and accessibility.

In conclusion, this study identified that accessibility by both the consulting and consultant teams was critical to interdisciplinary communication. These identified themes should be qualified and validated.

Twitter Julie Johnson @JulieJKJohnson and Anne M Stey @annemstey

Contributors Study design was performed by AMS and AL. Data collection and analysis was performed by AMS and PL. Data interpretation, writing and critical revision were performed by PL, AL, JLH, JJ, KYB and AMS.

Funding The authors have not declared a specific grant for this research from any funding agency in the public, commercial or not-for-profit sectors.

Competing interests None declared.

Patient consent for publication Not required.

Ethics approval Ethical approval for the observations and interviews were obtained from the Institutional Review Board of the University of California San Francisco who decided that this research was 'Not human subjects research' as no intervention was being performed and all observations and interviews were anonymous. IRB determined not human subjects research. Study ID 18-24556. Reference ID 213309.

Provenance and peer review Not commissioned; externally peer reviewed.

Data availability statement Data are available on reasonable request. Deidentified Interview transcripts are available on request by email to AMS.

Supplemental material This content has been supplied by the author(s). It has not been vetted by BMJ Publishing Group Limited (BMJ) and may not have been peer-reviewed. Any opinions or recommendations discussed are solely those of the author(s) and are not endorsed by BMJ. BMJ disclaims all liability and responsibility arising from any reliance placed on the content. Where the content includes any translated material, BMJ does not warrant the accuracy and reliability of the translations (including but not limited to local regulations, clinical guidelines, terminology, drug names and drug dosages), and is not responsible for any error and/or omissions arising from translation and adaptation or otherwise.

Open access This is an open access article distributed in accordance with the Creative Commons Attribution Non Commercial (CC BY-NC 4.0) license, which permits others to distribute, remix, adapt, build upon this work non-commercially, and license their derivative works on different terms, provided the original work is properly cited, appropriate credit is given, any changes made indicated, and the use is non-commercial. See: http://creativecommons.org/licenses/by-nc/4.0/.

\section{ORCID IDs}

Audrey Lyndon http://orcid.org/0000-0003-2215-4273

Anne M Stey http://orcid.org/0000-0002-8334-0304

\section{REFERENCES}

1 Davis JW, Hoyt DB, McArdle MS, et al. The significance of critical care errors in causing preventable death in trauma patients in a trauma system. J Trauma 1991;31:813-9.

2 Salas E, Wilson KA, Murphy CE, et al. Communicating, coordinating, and cooperating when lives depend on it: tips for teamwork. Jt Comm J Qual Patient Saf 2008;34:333-41.
3 Stey AM, Wybourn CA, Lyndon A, et al. How care decisions are made among interdisciplinary providers caring for critically injured patients: a qualitative study. Surgery 2020;167:335-9.

4 Hall P. Interprofessional teamwork: professional cultures as barriers. J Interprof Care 2005;19:188-96.

5 Weller J, Boyd M, Cumin D. Teams, tribes and patient safety: overcoming barriers to effective teamwork in healthcare. Postgrad Med J 2014;90:149-54.

6 Alvarez G, Coiera E. Interdisciplinary communication: an uncharted source of medical error? J Crit Care 2006;21:236-42.

7 Birkmeyer NJO, Finks JF, Greenberg CK, et al. Safety culture and complications after bariatric surgery. Ann Surg 2013;257:260-5.

8 Greenberg CC, Regenbogen SE, Studdert DM, et al. Patterns of communication breakdowns resulting in injury to surgical patients. J Am Coll Surg 2007;204:533-40.

9 Lingard L, Espin S, Whyte S, et al. Communication failures in the operating room: an observational classification of recurrent types and effects. Qual Saf Health Care 2004;13:330-4.

10 Shoham DA, Harris JK, Mundt M, et al. A network model of communication in an interprofessional team of healthcare professionals: a cross-sectional study of a burn unit. J Interprof Care 2016;30:661-7.

11 Teixeira C, Teixeira TM, Brodt SF. Appropriate medical professionals communication reduces intensive care unit mortality. Rev Bras Ter Intensiva 2010;22:112-7.

12 Leonard M, Graham S, Bonacum D. The human factor: the critical importance of effective teamwork and communication in providing safe care. Qual Saf Health Care 2004;13:i85-90.

13 Patton M. Qualitative research and evaluation methods. 3rd edn. Thousand Oaks: Sage Publications, 2002.

14 Braun V, Clarke V. Using thematic analysis in psychology. Qual Res Psychol 2006;3:77-101.

15 Braun V, Clarke V. Reflecting on reflexive thematic analysis. Qual Res Sport Exerc Health 2019;11:589-97.

16 Braun VC. To saturate or not to saturate? Questioning data saturation as a useful concept for thematic analysis and sample-size rationales. Qualit Res Sport Exer Health;2019:1-16.

17 Pannick S, Davis R, Ashrafian H, et al. Effects of interdisciplinary team care interventions on general medical wards: a systematic review. JAMA Intern Med 2015;175:1288-98.

18 O'Leary KJ, Johnson JK, Auerbach AD. Do interdisciplinary rounds improve patient outcomes? Only if they improve teamwork. J Hosp Med 2016;11:524-5.

19 Edmondson AC. Wicked problem Solvers. Harv Bus Rev 2016;94:117:52-9.

20 Bhamidipati VS, Elliott DJ, Justice EM, et al. Structure and outcomes of interdisciplinary rounds in hospitalized medicine patients: a systematic review and suggested taxonomy. J Hosp Med 2016;11:513-23.

21 Rosenstein $\mathrm{AH}$. Measuring and managing the economic impact of disruptive behaviors in the hospital. J Healthc Risk Manag 2010;30:20-6.

22 D'Lima DM, Murray EJ, Brett SJ. Perceptions of risk and safety in the ICU: a qualitative study of cognitive processes relating to staffing. Crit Care Med 2018;46:60-70.

23 Smith B. Generalizability in qualitative research: misunderstandings, opportunities and recommendations for the sport and exercise sciences. Qual Res Sport Exerc Health 2018;10:137-49.

24 Edwards BD, Day EA, Arthur W, et al. Relationships among team ability composition, team mental models, and team performance. $J$ Appl Psychol 2006;91:727-36.

25 Irvine R, Kerridge I, McPhee J, et al. Interprofessionalism and ethics: consensus or clash of cultures? J Interprof Care 2002;16:199-210. 